\title{
Os novos caminhos produtivos abertos pelas novas tecnologias do século XXI
}

\author{
The new productive paths opened by the technologies of the 21 st century
}

Anderson Elias Furtado

FARO - Faculdade de Roseira.

Erik Telles Pascoal

UNIFAL - Universidade Federal de Alfenas.

Kathleen Cristina Silveira Cunha

FARO - Faculdade de Roseira.

\begin{abstract}
The development of new manufacture technologies has contributed to the several changes discussed daily in the industrial scenario, such as industry 4.0 and production customization; and has also influenced important changes in commercial, social and employment relations. these innovative manufacturing tools include $3 \mathrm{~d}$ printing or DDM (direct digital manufacturing), nanotechnology, internet of things (IoT) and cyber physical systems among others. these new technologies have significantly impacted the timing and costs of large industries and, in addition, have open the path for the customization and sharing of the means of production among organizations. this article analyses these new technologies and the impacts they are generating in industry and in commercial and even personal relationships in today.
\end{abstract}

\section{RESUMO}

O desenvolvimento de novas tecnologias de manufatura tem contribuído para as diversas mudanças discutidas sistematicamente no cenário industrial, como a Indústria 4.0 e a customização da produção; e também tem influenciado importantes alterações nas relações industrial, comercial, social e de postos de trabalho. Essas ferramentas inovadoras de fabricação, incluem a impressão 3D ou DDM (Manufatura Digital Direta, da sigla em inglês), a nanotecnologia, as tecnologias de comunicação e informação (IoT) e sistemas cyber-físicos entre outras. Essas novas tecnologias têm impactado significativamente nos prazos e custos das grandes indústrias e, além disso, vem abrindo caminho para a customização e compartilhamento de meios de produção nas organizações. Esse artigo faz um balanço dessas novas tecnologias e dos impactos que elas estão gerando na indústria e nas relações comerciais e até pessoais na sociedade contemporânea.

\section{INTRODUÇÃO}

A sociedade está em constante evolução. Essa evolução pode ser quantificada de diversas formas.
Evidentemente, o nível de industrialização pode indicar o estado de avanço tecnológico de uma sociedade.

Ao primeiro grande salto tecnológico da sociedade moderna convencionou-se chamar Revolução Industrial. Ela se iniciou na Inglaterra do fim do século XVIII e marcou a transição dos métodos artesanais por máquinas $\mathrm{e}$ ferramentas. Foi também nessa época que surgiu a máquina a vapor e se intensificou a utilização do carvão, permitindo o crescimento das capacidades produtivas e de transporte [1].

Desde a Revolução Industrial, a indústria busca por soluções que visam melhorar seu desempenho. Esta busca impulsionou (e impulsiona) o desenvolvimento de várias tecnologias em todas as áreas relacionadas à produção. Ao longo do tempo é possível destacar algumas destas soluções e avanços tecnológicos, que foram adotados pela indústria e que representaram uma verdadeira ruptura na maneira como o sistema de produção era organizado, resultando em um aumento efetivo de produtividade, desempenho e, algumas vezes, constituindo um marco importante na evolução industrial, definindo as chamadas "revoluções" da indústria [2].

A primeira revolução no setor industrial é a do desenvolvimento das máquinas, já citada e que inicialmente chamou-se simplesmente Revolução Industrial. A próxima grande evolução estava ligada aos processos de fabricação e iniciou-se em meados do século XIX.

Em 1833, na Inglaterra - em um desenvolvimento despercebido - os primeiros passos em direção à produção industrial em massa estavam sendo feitos na produção de biscoitos para marinheiros. As linhas de embalagem de carne de Cincinnati da década de 1870 inspiraram o jovem Henry Ford a introduzir o princípio da produção de linhas de montagem no mundo dos carros - que na época ainda eram produzidos artesanalmente. O primeiro veículo Ford saiu da linha de montagem em 1913 e essa nova forma de organização e produção seriada tomou conta de todo o mundo. [3]. 
Os elementos característicos da primeira Revolução Industrial como o ferro, o carvão e a energia a vapor, agora dão lugar aos novos elementos representantes da segunda Revolução Industrial: o aço, a eletricidade e o petróleo.

Nas décadas de 1950 e 1970, começou-se a desenhar aquela que viria a ser considerada a terceira Revolução Industrial, a Revolução Eletrônica ou Digital, com a proliferação e uso dos semicondutores, dos computadores, automação e robotização em linhas de produção, com informação armazenada e processada de forma digital, as comunicações, os telefones móveis e a internet [4].

Atualmente, observa-se o desenvolvimento da quarta Revolução Industrial, denominada como Indústria 4.0, sendo a digitalização da operação industrial. Este contexto se originou através de um projeto de empresas, universidades e do governo alemão, sendo citado pela primeira vez durante a Hannover Fair, em 2011, com o intuito modernizar as indústrias locais. Sendo assim, o termo Indústria 4.0 promove a informatização da indústria e tem como base as inovações tecnológicas, permitindo a conectividade da automação, do controle e da tecnologia da informação para aprimorar os processos da manufatura e sua eficiência. O objetivo deste contexto é criar as fábricas inteligentes (Smart Factory), no qual, o processo de produção será totalmente digitalizado e conectado em rede através de sistemas de informação, tornando a produção autônoma e inteligente [5].

$\mathrm{Na}$ Figura 1, encontra-se uma representação esquemática da evolução histórica da produção industrial no mundo.

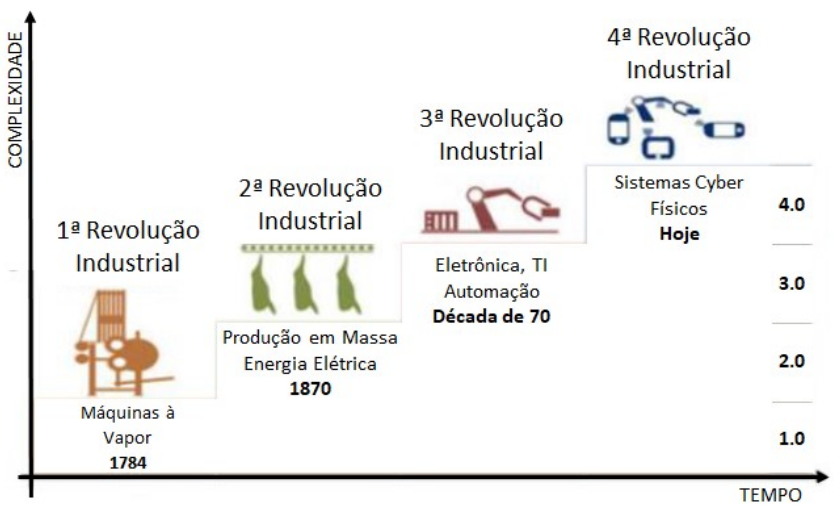

Figura 1: Evolução histórica da produção industrial no mundo. Fonte: [3] [4].

Esse artigo busca fazer um balanço das novas ferramentas inovadoras de fabricação de materiais que estão suportando a construção da Indústria 4.0 como a impressão 3D, a nanotecnologia, a prototipagem rápida e DDM (Direct Digital Manufacturing) e dos seus impactos não somente no cenário industrial como também nas relações comercial, social e de postos de trabalho.
Para tanto, esse estudo está organizado da seguinte forma. Além desta introdução, a seção 2 apresenta uma revisão da literatura, explicando os temas da Indústria 4.0 e das novas tecnologias e ferramentas de manufatura. A seção 3 apresenta as discussões e os resultados sobre os impactos da utilização das novas ferramentas de manufatura tanto no cenário industrial como também nas relações comercial, social e de postos de trabalho. Por fim, na seção 4, apresenta-se a conclusão do artigo e sugestões de futuros trabalhos.

\section{REVISÃO DA LITERATURA}

Esta seção está dividida em duas subseções. $\mathrm{Na}$ primeira subseção é descrita a Indústria 4.0 no mundo, e a segunda subseção aborda as novas ferramentas inovadoras de manufatura.

A INDÚSTRIA 4.0 NO MUNDO: O setor de manufatura industrial continua sendo crucial para o crescimento e aumento dos empregos pelo mundo. Para exemplificar essa importância, na Europa Ocidental, mesmo se apenas uma em cada dez empresas pode ser classificada como indústria de manufatura (essa classificação exclui os setores de mineração, construção e energia), o setor compreendia em 2015, dois milhões de empresas e 33 milhões de empregos diretos. Soma-se a isso o fato de cada novo emprego criado na indústria resultar na criação de meio a dois empregos em outros setores. Apesar disso, a participação da indústria na economia europeia e mundial vem declinando. A Europa perdeu um terço de seu parque industrial nos últimos 40 anos, boa parte disso, em função do surgimento da manufatura em outras partes do mundo, destacando-se a China [6]. A fim de conter esse declínio, desde o início da década atual, os países do primeiro mundo vêm estudando o que seria a indústria do ano de 2020

Nos Estados Unidos, foi criado em 2011 o projeto Advanced Manufacturing Partnrship a fim de unir a academia, indústria e governo na busca da revitalização da manufatura no país [7].

O termo Indústria 4.0 surgiu na Alemanha, em março de 2012, como um projeto do governo cujo objetivo estratégico foi o de explorar o alto potencial econômico e de inovação resultante do impacto das tecnologias de informação e comunicação na indústria [8].

Em 2013, o governo francês lançou os seus "34 plans de reconquête industrielle". Nesse plano, a indústria do futuro (também chamada de Smart factory, usine numérique ou I'industrie 4.0) é descrita como um dos meios de relançar a indústria francesa [9].

Na China, o plano Made in China 2025 foi redigido em 2015 pelo ministério da Indústria e Tecnologia da Informação em parceria com a Academia Chinesa de Engenharia e inspirado diretamente pela iniciativa alemã Industrie 4.0 [8]. 
Ainda hoje, a Quarta Revolução Industrial ou Indústria 4.0 é um termo não muito preciso usado em referência a uma variedade de mudanças tecnológicas e inovações que vem ocorrendo desde o início do século XXI, com efeitos potencialmente dramáticos na economia e sociedade. Ela se caracteriza por uma automação crescente, sobretudo nos trabalhos de baixa e média variabilidade, aumento da conectividade, aprendizado de máquina (machine learning no termo em inglês amplamente utilizado no Brasil) e desenvolvimento de novas tecnologias, todos ocorrendo numa velocidade consideravelmente maior do que as revoluções industriais precedentes [10].

\section{AS NOVAS TECNOLOGIAS E}

FERRAMENTAS DE MANUFATURA: Nesta seção são apresentadas as principais características das novas ferramentas de manufaturas.

Direct Digital Manufacturing, Impressão 3D ou manufatura aditiva: Os processos de manufatura podem ser divididos em três grandes grupos: os processos por subtração, por conformação e por adição. A diferença de conceito entre eles pode ser vista na Figura 2.

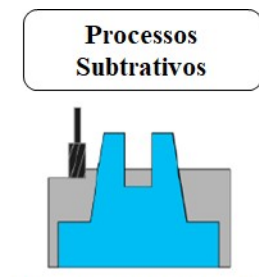

- Semi-acabados

- Utilização de

ferramentas

- CAD-CAM opciona
- Remoção de material
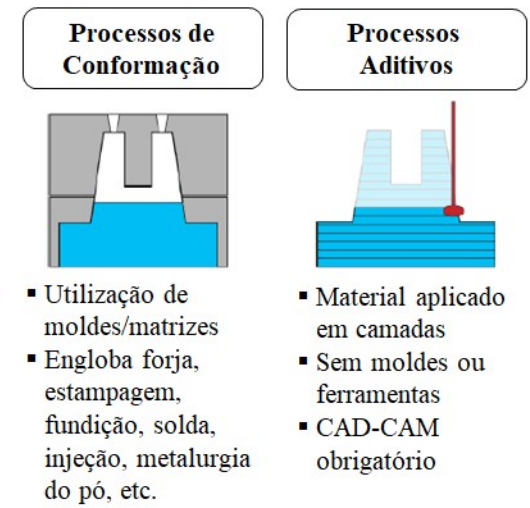

Figura 2: Os diferentes processos de manufatura e suas características. Fonte: [11].

Podemos distinguir três tipos principais de fabricação aditiva: (1) a prototipagem rápida (confecção de peças protótipo), (2) a construção de ferramentais em soft tooling (realização de moldes ou matrizes de forja, por exemplo) e (3) a fabricação direta (fabricação de peças diretamente de arquivos numéricos e que respondam diretamente às características funcionais esperadas do produto final) [12].

A origem da manufatura digital direta ou manufatura aditiva foi a "prototipagem rápida", usada predominantemente na construção de maquetes de design no fim dos anos 1980. Hoje, há usos disseminados não só em prototipagem, mas também na fabricação de ferramentas e manufatura de pequeno volume em diversos setores da indústria [13].

Os processos aditivos foram iniciados com polímeros pela sua facilidade de adaptação à tecnologia. Mais recentemente, o alcance da tecnologia foi bastante ampliado com a adaptação da tecnologia para componentes metálicos.

A fabricação aditiva metálica agrupa diversos processos cuja produtividade e níveis de maturidade são variáveis. Assim, os materiais divergem segundo a tecnologia aplicada, a peça a fabricar e sua utilização. Hoje esses processos se encontram principalmente nas indústrias de alto valor agregado (espacial, aeronáutica e médica), especialmente com materiais exóticos (titânio, inconel, etc.) [14].

Podemos dividir a fabricação aditiva a base de pós-metálicos em três grandes famílias de processos: na primeira não há fusão do pó metálico (aquela que conhecemos como impressão 3D), e duas com fusão do pó metálico: uma por aporte direto e outra por cama de pó. Nesses casos pode-se ter a fusão por EBM (feixe de elétrons) ou SLM (laser) [14].

Durante a fabricação, peças metálicas construídas por manufatura aditiva experimentam um histórico complexo de variações térmicas, envolvendo extração térmica direcional, repetidas fundições e solidificação rápida. Muitas ligas ainda passam por diversas transformações de fases no estado sólido. Esses fatores tornam a análise da evolução microestrutural complexa e induzem a propriedades mecânicas que não são tipicamente encontradas nos processos de fabricação convencional. Entretanto, em geral, as propriedades mecânicas estáticas dos componentes metálicos produzidos por manufatura aditiva são comparáveis às daqueles produzidos em processos convencionais [15].

A manufatura aditiva de metais carrega em si o potencial de redução da pegada de carbono por meio da otimização do projeto e a redução de perda de material. O projeto ATIKINS, realizado em 2007, concluiu que um design otimizado pode representar uma redução de peso e material em torno de $40 \%$ [15].

Nanotecnologia: A nanotecnologia é o estudo da manipulação da matéria em escala atômica e molecular. Em termos matemáticos um nanômetro $(\mathrm{nm})$ correspondente à bilionésima parte de um metro $\left(1 \mathrm{~nm}=10^{-9} \mathrm{~m}\right)$ [16] [17].

Entretanto, a nanotecnologia não é uma ciência nova. Há muitas décadas os químicos já sintetizam polímeros (grandes cadeias de moléculas feitas de minúsculas unidades nano escalares) e também as nanopartículas ocorrem naturalmente no leite, combustão e cozimento. Mas, somente, em 1974, o professor Norio Taniguchi, da Universidade de Ciência de Tóquio cria a palavra "nanotecnologia", significando máquinas com tolerância de menos de um micro [16] [17].

E mais recentemente equipamentos sofisticados foram desenvolvidos para manipular matéria em escala manométrica como a Microscopia de Varredura por Tunelamento (STM - Scanning Tunneling Microscopy), em 
1981 e a Microscopia de Força Atômica (AFM - Atomic Force Microscopy), em 1986 [16] [17].

O objetivo da nanotecnologia é criar, caracterizar, produzir e aplicar estruturas, dispositivos e sistemas, controlando a forma e o tamanho na escala manométrica. $\mathrm{E}$ porque manipular átomos e moléculas? Dentre outras razões pode-se destacar: curiosidade científica, dimensões físicas de dispositivos ULSI (Ultra Large Scale Integration), novos materiais, novos dispositivos, nova eletrônica, produtos mais eficientes e incontáveis utilizações em diversas áreas como a medicina, eletrônica, ciência da computação, física, química, biologia e engenharia dos materiais entre outras, conforme ilustrado na Figura 3 [16].

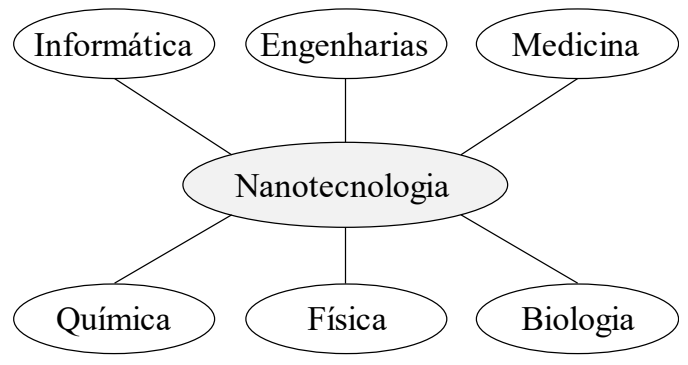

Figura 3: Algumas áreas de aplicação da nanotecnologia. Fonte: [16].

A nanotecnologia possui um enorme potencial de aplicação, tais como: aumentar a capacidade de armazenamento e processamento de dados dos computadores, criar novos mecanismos para a produção de medicamentos, criar materiais mais leves, baratos e mais resistentes, economia de energia, proteção ao meio ambiente, menor uso de matérias-primas escassas [16]. Atualmente os chamados "nanoprodutos" estão presentes nos setores mais relevantes da economia global, como exemplificado na Tabela 1. De acordo com estimativas, mais de 12 mil empresas em 53 países no mundo, já produzem os "nanoprodutos", cujo faturamento previsto para 2020 é superior a US\$ 3 trilhões [18].

Tabela 1: Exemplos de nanoprodutos. Fonte: [18].

\begin{tabular}{|l|l|}
\hline \multicolumn{1}{|c|}{ Setores } & \multicolumn{1}{c|}{ Nanoprodutos } \\
\hline $\begin{array}{l}\text { Saúde/ } \\
\text { Medicina }\end{array}$ & $\begin{array}{l}\text { Produção UV em revestimentos cosméticos, } \\
\text { agentes de contrastes, tratamento de tumor, } \\
\text { diagnóstico in vitro, etc. }\end{array}$ \\
\hline Energia & $\begin{array}{l}\text { Baterias solares e fotovoltaicas, ânodos de } \\
\text { bateria de lítio, etc. }\end{array}$ \\
\hline Eletrônicos & $\begin{array}{l}\text { Painéis (LED/OLED), tintas para impressão } \\
\text { eletrônica, ferrofluidos, } \\
\text { semicondutores/isolantes, etc. }\end{array}$ \\
\hline Indústria & $\begin{array}{l}\text { Nanofluídos, aditivos, fotocatálise, materiais } \\
\text { de polimento, cerâmica, etc. }\end{array}$ \\
\hline $\begin{array}{l}\text { Agro/ } \\
\text { Ambiente }\end{array}$ & $\begin{array}{l}\text { Tratamento de água, agroquímicos e } \\
\text { fertilizantes, remoção de poluentes, etc. }\end{array}$ \\
\hline Outros & $\begin{array}{l}\text { Produtos auto-limpantes, aplicação de } \\
\text { pigmentos, plástico/embalagem antiestática, } \\
\text { etc. }\end{array}$ \\
\hline
\end{tabular}

As iniciativas nacionais em nanotecnologia iniciaram em 2002 por meio das Redes Cooperativas em Nanociências \& Nanotecnologias, financiadas pelo CNPq. O fruto deste trabalho resultou na criação em 2013 da Iniciativa Brasileira em Nanotecnologia (IBN) que é atualmente o principal programa estratégico para incentivo da nanotecnologia no Brasil [18].

Um dos pilares do IBN é o SisNANO (Sistema Nacional de Laboratórios em Nanotecnologias), que é um sistema de laboratórios direcionados à pesquisa, desenvolvimento e inovação (PD\&I) em nanociências e nanotecnologia, tendo como característica essencial o caráter multiusuário e de acesso aberto. O laboratório de referência do SisNANO é Laboratório Nacional de Nanotecnologia (LNNano), criado em 2011 em CampinasSP [18].

IoT - Internet das Coisas (Internet of Things, no original em Inglês): Atualmente, a internet se transforma sucessivamente em uma "hiper-rede", formada por diversas conexões entre equipamentos (físicos e documentais), atores (biológicos ou algoritmos) e conceitos (informação por links e metadados), chamada Internet dos objetos ou Internet das Coisas. Essa rede possibilita a conexão de bilhões de seres humanos e a interação entre objetos. Dessa forma, ela se caracteriza como a ferramenta mais potente gerada pelo homem, para criar, modificar e compartilhar informações em todo o mundo [19].

Levando em consideração todo o potencial proporcionado pela internet, Kevin Ashoton, cofundador e diretor executivo do Auto-ID no Institute of Technology (MIT), analisou os benefícios que a mesma seria capaz de agregar ao cenário industrial da época, e com isso no ano de 1999, apresentou ao mundo o termo "Internet of Things", como uma oportunidade de se etiquetar eletronicamente os produtos da linha de produção, inicialmente utilizando uma infraestrutura de Radio Frequency Indentification (RFID) [20]. Contudo, em 2005, o termo altamente procurado e que representa relação com a IoT foi o Wirelless Sensor Network (WNS), em português Redes de Sensores Sem Fio (RSSF), a qual é diretamente responsável pelos avanços em automação industrial e residencial [19].

Existem ainda outros sistemas. Entre eles, o NFC (Near field communication) - que pode ser considerado a integração do RFID aos telefones celulares, M2M (Machine to Machine Communication) e $\mathrm{V} 2 \mathrm{~V}$ (Vehicle to Vehicle Communication) [21].

Em outras palavras, a IoT representa uma extensão da Internet atual e permite aos objetos com capacidade computacional e da comunicação se conectarem a internet. Assim, essa conexão permite a nós usuários controlar 
objetos remotamente ou torná-los provedores de serviços [22].

No contexto da Indústria 4.0, a IoT, comumente mencionada como Internet Industrial das Coisas (IIoT), é responsável pela interligação direta entre dispositivos que utilizam sinais a partir de sensores, dispositivos de controles integrados, dados de armazenamento em nuvem e códigos específicos de usuários, possibilitando um alto grau de automação remota; e proporciona que máquinas e equipamentos sejam capazes de coletarem dados em tempo real dos ambientes que os cercam [23].

Desta forma, a IIoT proporciona o aumento da confiabilidade no processo produtivo, serve como base para um novo nível de organização e gerenciamento de cadeias de valor industriais e permite a flexibilidade produtiva e de economia de recursos, como a redução de custos em produtos e prestações de serviços [24].

Sistemas cyber-físicos (CPS): Os sistemas cyberfísicos (CPS - na sigla em inglês) consistem na associação entre computação e processos físicos de fabricação, que compreendem máquinas inteligentes, sistemas de armazenamentos e instalações de produção que foram desenvolvidas digitalmente. Os computadores e redes incorporadas são responsáveis por monitorar e controlar os processos físicos, normalmente com ciclos de feedback nos quais as fases do processo de fabricação criam sinais de retorno para realimentar o sistema de computadores que gerenciam um processo. A descentralização e comportamentos autônomo de processo de produção são as principais características dos CPS [25].

Os CPSs utilizam elementos de computação para coordenarem e se comunicarem com sensores, que são encarregados de monitorarem indicadores virtuais e físicos, e os atuadores, que modificam o ambiente virtual e físico em que são executados [26]. Na Figura 4, é exemplificado um esquema do funcionamento CPS.



Figura 4: Funcionamento do CPS. Fonte: [27].
Para a utilização dos CPSs no setor de manufatura é fundamental desenvolvimentos complementares, como uma abordagem sistemática, baseada em métodos de agrupamento adaptável para máquinas, a fim de levar a inteligência ao chão de fábrica e fornecer produção continua e tempo de inatividade quase zero. Assim, a arquitetura CPS de cinco níveis, denominada 5C, constituise de metodologias e orientações para a introdução do CPS para o ramo de fabricação, abrangendo desde a fase de aquisição dos dados até a análise e criação de valor final [28] [29], como pode ser visualizado na Figura 5.

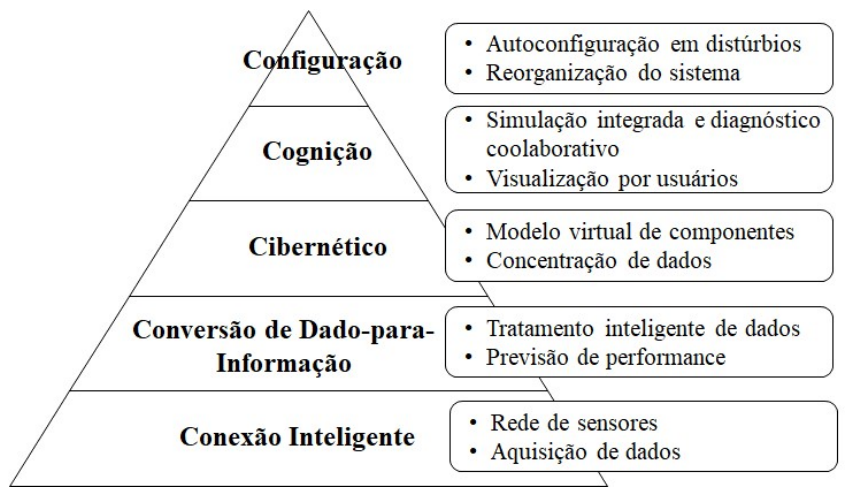

Figura 5: Arquitetura 5C. Fonte: [26] [29].

- Conexão inteligente: Tem a função de realizar a obtenção de dados de máquinas e seus componentes.

- Conversão de dados para informação: Trata os dados adquiridos na camada inferior, convertendoos em informações analisáveis para a obter conclusões.

- Cibernética: Opera como uma central de informações, obtendo os dados de todas as máquinas conectadas e elaborando um modelo virtual do sistema.

- Cognitiva: Neste nível ocorre a formatação dos resultados da camada inferior para a apresentação ao pessoal qualificado.

- Configuração: Essa camada do modelo serve como um retorno do ambiente virtual para o físico, atuando como um sistema de controle e supervisório.

$\mathrm{Na}$ infraestrutura do sistema produção introduzido para a Indústria 4.0, o CPS pode melhorar os processos de informações em tempo real entre máquinas industriais, as quais também podem registrar-se na rede e trocar informações por meio de interfaces; cadeias de fornecimento de fabricação, provendo maior grau de visibilidade e controle sobre as cadeias de fornecimento, aprimorando a rastreabilidade e a segurança de mercadorias; sistemas de negócios e fornecedores. 
Nesta seção são analisados os seus impactos no contexto industrial, comercial, social, postos de trabalho e desafios para cada uma das tecnologias e ferramentas de manufatura.

$\mathrm{Na}$ Tabela 2, são apresentados os impactos principais da manufatura aditiva.

Tabela 2: Implicações da manufatura aditiva. Fonte: Autores.

\begin{tabular}{|c|c|}
\hline \multicolumn{2}{|r|}{ Impactos } \\
\hline $\begin{array}{l}\text { Cenário } \\
\text { industrial }\end{array}$ & $\begin{array}{l}\text { A manufatura aditiva muda o paradigma da } \\
\text { produção. Tradicionalmente, a fabricação } \\
\text { de peças ocorre pela retirada de material. } \\
\text { Na fabricação aditiva, a produção acontece } \\
\text { pela adição de material, o que muda } \\
\text { fundamentalmente os métodos de } \\
\text { concepção e abre novas perspectivas tanto } \\
\text { no domínio da aplicação quando da } \\
\text { fabricação de novas formas. Assim, a } \\
\text { manufatura aditiva (também conhecida } \\
\text { como impressão 3D) é um conjunto de } \\
\text { processos que permite fabricar, camada a } \\
\text { camada, um objeto físico a partir de um } \\
\text { modelo numérico [30]. }\end{array}$ \\
\hline $\begin{array}{l}\text { Relações } \\
\text { comerciais }\end{array}$ & $\begin{array}{l}\text { Entretanto, a tecnologia não é totalmente } \\
\text { perfeita e aparecem defeitos inerentes ao } \\
\text { processo: as superfícies, por exemplo, } \\
\text { aparecem bastante rugosas ao fim da } \\
\text { produção. Desta forma, é bom lembrar que, } \\
\text { apesar de se apresentar como uma } \\
\text { alternativa aos modelos de usinagem } \\
\text { clássica, a fabricação aditiva } \\
\text { provavelmente não substituirá os processos } \\
\text { clássicos, mas sim será complementar nas } \\
\text { pequenas séries e produção e na fabricação } \\
\text { de peças de grande valor agregado [31]. }\end{array}$ \\
\hline $\begin{array}{l}\text { Relações } \\
\text { sociais e } \\
\text { Postos de } \\
\text { trabalho }\end{array}$ & $\begin{array}{l}\text { A fabricação aditiva oferece a vantagem de } \\
\text { se desvencilhar das limitações dos } \\
\text { processos de conformação e usinagem } \\
\text { clássicos. Isso permite aos projetistas } \\
\text { focarem somente nas necessidades reais de } \\
\text { utilização, possibilitando, por vezes, a } \\
\text { substituição de diversos componentes em } \\
\text { um conjunto por apenas um monobloco. } \\
\text { Assim, pode-se obter um ganho em } \\
\text { produtividade em termos de peso, tempo e } \\
\text { custo [31]. }\end{array}$ \\
\hline
\end{tabular}

Na Tabela 3, são apresentados os impactos principais da Nanotecnologia.
Tabela 3: Implicações da nanotecnologia. Fonte: Autores. Impactos

Cenário São previstos impactos nas cadeias industrial produtivas dos diversos setores industriais e modificações dos processos produtivos atuais de forma a fabricar produtos:

- Mais eficazes e precisos,

- Que consomem menos insumos e energias,

- Que agreguem novas ou diferenciadas funcionalidades a produtos já existentes,

- Que contribuam para a otimização dos custos: desde a concepção à produção [18],

Promoção de incentivos à pesquisa e desenvolvimento (P\&D) multidisciplinar e interdisciplinar com um potencial capaz de revolucionar os métodos atuais nos quais materiais e produtos são criados [32].

Desenvolvimento da indústria militar e aeroespacial [18].

Relações O desenvolvimento das nanotecnologias comerciais contribuirá para a:

- Criação de novas empresas,

- Estabelecimento de parcerias comerciais entre empresas e centros tecnológicos,

- Geração de novas patentes [18].

Segundo estimativas a nanotecnologia está presente em produtos de mais de 12 mil empresas de 53 países, cujo faturamento global estimado para 2020 é de aproximadamente US \$ 3 trilhões [18].

Relações A nanotecnologia deverá promover:

sociais e $\quad \circ \quad$ A transição do atual modelo

Postos de econômico (embasado no consumo)

trabalho para uma economia sustentável (fundamentada em eficiência de ciclo de vida de produtos e processos),

- A geração de empregos e renda em novas áreas, mas outros postos de trabalho poderão desaparecer,

- A geração de riquezas para os países.

Por outro lado, ainda existem vários questionamentos sobre a aplicabilidade da nanotecnologia, especialmente aos riscos à saúde e ao meio ambiente (nanotoxicologia), ao ciclo produtivo (nanossegurança), aos aspectos éticos, à responsabilidade social corporativa, aos aspectos regulatórios e de segurança mundial (nanoterrorismo) [16] [18].

$\mathrm{Na}$ Tabela 4, são apresentados os impactos principais da IoT. 
Tabela 4: Implicações da IoT. Fonte: Autores.

\section{Impactos}

Cenário No ambiente industrial o processo de

industrial comunicação entre os mundos físicos e digital é conhecido como "Internet Industrial das Coisas (IIoT)". Sua função é criar uma rede inteligente entre máquinas, propriedades, sistemas de comunicação, produtos inteligentes e indivíduos em toda a cadeia de valor da empresa, durante todo o ciclo de vida do produto.

A partir dessas informações disponibilizadas nesta rede, processos e contratos podem ser coordenados com o objetivo de aumentar a eficiência da empresa, otimizar o tempo de produção e de logística, reduzir energia, aumentar a qualidade dos produtos [20].

Relações A IoT proporciona novos modelos de comerciais negócios, trazendo inúmeros benefícios para as empresas e população, tais como:

- Transporte (informações em tempo real sobre as melhores rotas, etc.),

- Comércio (produção automática de acordo com a demanda do estabelecimento comercial, etc.),

- Saúde (acompanhamento médico em tempo real etc.),

- Serviços públicos (sensores para alerta de enchentes, etc.)

- Agronegócio (estações agrometereológicas etc.),

- Comunicação (bluetooth, etc.) [20].

Relações

sociais e

Postos de A IoT no cotidiano possibilita a automação de trabalho objetos e equipamentos domésticos, como: portão automático, controle de temperatura e iluminação ou até uma casa totalmente automatizada [24].

Na Tabela 5, são apresentados os impactos principais dos sistemas cyber-físicos.
Tabela 5: Implicações dos sistemas cyber-físicos. Fonte: Autores.

\begin{tabular}{|c|c|}
\hline \multicolumn{2}{|r|}{ Impactos } \\
\hline $\begin{array}{l}\text { Cenário } \\
\text { industrial }\end{array}$ & $\begin{array}{l}\text { O sistema cyber-físico introduz no setor } \\
\text { industrial por meio de uma estrutura em cinco } \\
\text { níveis, agregação de máquinas } \\
\text { equipamentos, por meio de um modelo } \\
\text { virtual, cujo objetivo levar a inteligência ao } \\
\text { chão de fábrica, reduzir o tempo de parada, } \\
\text { analisar e tratar os dados para tomada de } \\
\text { decisões [29]. }\end{array}$ \\
\hline $\begin{array}{l}\text { Relações } \\
\text { comerciais }\end{array}$ & $\begin{array}{l}\text { Os sistemas cyber-físicos não implicam } \\
\text { necessariamente em novos modelos de } \\
\text { negócios. Por tratarem da integração de } \\
\text { atuadores em resposta a sensores, controlados } \\
\text { por IA, terão muito mais impacto dentro do } \\
\text { cenário industrial, sobretudo em relação às } \\
\text { formas de trabalho. }\end{array}$ \\
\hline $\begin{array}{l}\text { Relações } \\
\text { sociais e } \\
\text { Postos de } \\
\text { trabalho }\end{array}$ & $\begin{array}{l}\text { A popularização dos sistemas cyber-físicos, } \\
\text { sobretudo funcionando em conjunto com a } \\
\text { Internet das coisas, ampliará a automatização } \\
\text { das análises e até decisões menos sofisticadas, } \\
\text { impactando fortemente não só na mão de obra } \\
\text { fabril, como também nas funções suporte, } \\
\text { como engenharia de processos e produção. Os } \\
\text { postos de trabalho e funções, se não } \\
\text { suprimidos, ao menos mudarão drasticamente } \\
{[28] \text {. }}\end{array}$ \\
\hline
\end{tabular}

\section{CONCLUSÃO}

O desenvolvimento e aplicação das novas tecnologias de manufatura é uma realidade global inevitável. Por meio delas, as máquinas serão cada vez mais automatizadas e inteligentes. Os processos de produção continuarão se modernizando e proporcionando a criação de novos produtos.

Apesar dos diferentes níveis de impacto que cada uma das tecnologias ocasionará, pode-se verificar que sua popularização modificará profundamente não só as relações industriais, mas também as relações sociais e comerciais.

No quesito das relações industriais, destaca-se que a utilização das novas tecnologias permitirá a modernização dos processos produtivos e, com isso, contribuirá para a redução dos custos industriais. Por meio de máquinas mais automatizadas e inteligentes será possível ter uma maior flexibilidade de produção e consequente customização dos produtos. Estes elementos somados a uma estratégica coerente de identificação de demandas e tendências permitirá às organizações industriais acelerar a colocação de novos produtos no mercado, aumentando assim a sua competitividade.

No que tange às relações comerciais, a oportunidade de criação de novos modelos de negócios e 
mercados revela-se com o principal ganho vinculado à aplicação das novas tecnologias.

É no âmbito das relações sociais que residem as principais preocupações a respeito da aplicação das novas tecnologias. Pode-se citar: regularização dos aspectos legais (nanotecnologia), cyber-segurança (quanto mais conectada uma organização estiver mais sujeita ela ficará de uma espionagem), utilização para fins ilícitos (nanoterrorismo) e questões ambientais.

Mas nenhuma dessas questões, supramencionadas, preocupa tanto quanto os inevitáveis impactos das novas tecnologias no mercado de trabalho. Muitos postos de trabalho serão inevitavelmente suprimidos. Quanto menos estratégico for um posto maior será a possibilidade de ele ser suprimido.

Por outro lado, serão criados muitos postos em setores e funções ligados a esses novos paradigmas. Podese citar as áreas de análise de dados, como cientistas de dados, desenvolvimento de Softwares e Inteligência Artificial. Outros tantos postos e funções serão modificados ou realocados para atividades estratégicas.

Analisando intrinsicamente as novas tecnologias, um fator que chama atenção é a interrelação e complementariedade entre elas. A IoT, por exemplo, se mostra fundamental para um funcionamento ótimo dos sistemas cyber-físicos. Já a existência de sistemas cyberfísicos bem estruturados pode aprimorar significativamente a eficiência dos processos de manufatura aditiva. E assim, este aspecto se repete sucessivamente entre as demais tecnologias e ferramentas. Isso indica que a utilização das novas tecnologias e ferramentas não ocorrerá de forma isolada, e sim que, para um resultado eficaz, existirá uma interdependência entre elas.

Por fim, pode-se concluir que essas tecnologias mudarão profundamente a forma como nos relacionamos no trabalho e, em último caso, serão fonte de mudança na nossa relação social e até educacional, já que novas formas de aprendizado serão necessárias para atender às novas demandas de profissões que melhor se adequarão a essa nova forma de produzir.

\section{REFERÊNCIAS}

[1] BANZATO, E. "Indústria 4.0. Com a IoT (Internet of Things) se consolida mais uma nova revolução industrial, que demanda tecnologia e mudança no modelo de negócio". Revista LOGÍSTICA, agosto de $2015 . \quad$ Disponível em: $<$ https:/www.imam.com.br/logistica/artigos/serietecnologia-da-informacao/2278-industry-4.0>. Acesso em: novembro de 2019.

[2] SUGAYAMA, R.; NEGRELLI, E. "Veículo conectado na rota da Indústria 4.0". In: Anais do XXIV Simpósio Internacional de Engenharia Automotiva - SIMEA 2016, p. 48-63. São Paulo: Blücher, 2016. ISSN 2357-7592, DOI 10.5151/engpro-simea2016-PAP16.

[3] BARTEVYAN, L. "DLG-Expert report 5/2015: Industry 4.0 - Summary report", p. 1-8. Disponível em: $<$ https://www.cenit.com/fileadmin/dam/Corporate/PDFs/20 15_5_Expertenwissen_E.pdf $>$. Acesso em: abril de 2020.

[4] COSTA, C. "Indústria 4.0: O futuro da indústria nacional". POSGERE - Revista para Pós-Graduandos (ISSN 2526-4982). p. 5-14. V. 1, n. 4, setembro de 2017.

[5] OLIVEIRA, F. T.; SIMÕES, W. L. “A Indústria 4.0 e a produção no contexto dos estudantes de engenharia”. In Anais do I SIENPRO-2017 - ISSN 2594-410X. Disponível em:

$<$ https://sienpro.catalao.ufg.br/up/1012/o/Fernanda_Tha $\%$ C 3\%ADs_de_Oliveira.pdf $>$. Acesso em: dezembro de 2019.

[6] DAVIES, R. "Industry 4.0 - Digitalisation for productivity and growth". EPRS - European Parliamentary Research Service, Breafin, setembro de $2015 . \quad$ Disponível em: $<$ https://www.europarl.europa.eu/RegData/etudes/BRIE/20 15/568337/EPRS_BRI(2015)568337_EN.pdf>. Acesso em: janeiro de 2020 .

[7] THE WHITE HOUSE, "President Obama launches advanced manufacturing partnership steering committee “2.0". Disponível em: $<$ https://obamawhitehouse.archives.gov/the-pressoffice/2013/09/26/president-obama-launches-advancedmanufacturing-partnership-steering-com $>$. Setembro de 2013. Acesso em: novembro de 2019.

[8] ASSAD NETO, A.; PEREIRA, G. B.; DROZDA, F. O.; SANTOS, A. P. L. "A busca de uma identidade para a indústria 4.0". Brazilian Journal of Development, v. 4, n. 4, p. 1379-1395. ISSN : 2525-8761. Curitiba, Jul/set de 2018. Disponível em: $<$ http://www.brazilianjournals.com/index.php/BRJD/article/ view/183/153>. Acesso em: fevereiro 2020. 
[9] RICHARD, P. "L’Usine 4.0, c'est quoi ?". Techniques de l'ingenieur. 26 de outubro de 2016. Disponível em: $<$ https://www.techniques-

ingenieur.fr/actualite/articles/lusine-4-0-cest-quoi-37271/>. Acesso em: dezembro de 2019.

[10] WARD, M. "Fourth industrial revolution". Debate pack, CDP 2016/0153. The House of Commons Library. Londres. Setembro de 2016. Disponível em: < https://commonslibrary.parliament.uk/researchbriefings/cdp-2016-0153/>. Acesso em: março 2020.

[11] TRONCO, M. L. "Manufatura Aditiva". Aula EESC-USP. Disponível em: $<$ https://edisciplinas.usp.br/pluginfile.php/3459951/mod_re source/content/1/Aula_Manufatura_Aditiva.pdf $>$. Acesso em : dezembro 2019.

[12] POUZET, S. "Fabrication additive de composites à matrice titane par fusion laser de poudre projetée." 199f. Tese de Doutorado - École Nationale Supérieure d'Arts et Métiers, França, 2015. Disponível em : $<$ https://pastel.archives-ouvertes.fr/tel-

01315553/document>. Acesso em : janeiro 2020.

[13] HOLMWTRÖM, J. ; HOLWEG, M.; KHAJAVI, S.; PARTANEN, J. "The direct digital manufacturing (r)evolution: Definition of a research agenda." Springer, 18 de janeiro de 2016. DOI 10.1007/s12063-016-0106-z.

[14] CALVES, P. "Les métaux pour la fabrication additive". Industrie e Technologies, n 991. Outubro de 2016.

[15] FRAZIER, W. E. "Metal Additive Manufacturing: A review" Jounal of Materials Engineering and Performance. Abril de 2014. DOI : 10.1007/s11665-0140958-z.

[16] PACHECO, M. A. "Uma Introdução à Nanotecnologia" 2019. ICA: Laboratório de Inteligência Computacional Aplicada. PUC-Rio. Disponível em: $<$ https://docero.com.br/doc/vevecn $>$. Acesso em: abril de 2020 .

[17] CADIOLI, L. P., SALLA, L. D. "Nanotecnologia: um estudo sobre seu histórico, definição e principais aplicações desta inovadora tecnologia" 2006. Revista de Ciências Exatas e Tecnologia, v.1, n.1. DOI: 10.17921/1890-1793.2006v1n1p98-105.

[18] CENTRO NACIONAL DE PESQUISA EM ENERGIA E MATERIAIS (CNPEM). 2019 "Benefícios e riscos das nanotecnologias". Disponível em: $<$ http://cnpem.br/wpcontent/uploads/2019/10/SEPARATA-CNPEM02 Benef $\%$ C $3 \%$ ADcios-e-riscos-dasnanotecnologias.pdf $>$. Acesso em: abril de 2020.
[19] SALEH, I. "Les enjeux et les défis de l'internet des Objets (IdO)". ISTE Ltd. London, UK - openscience.fr, $2017 . \quad$ Disponível em: $<$ https://www.openscience.fr/IMG/pdf/iste_idov1n1_1.pdf $>$ Acesso em: janeiro 2020.

[20] ASHTON, K. "That 'Internet of Things' things". RFiD Journal, 2009. Disponível em: $<$ http://www.itrco.jp/libraries/RFIDjournal-

That $\% 20$ Internet $\% 20$ of $\% 20$ Things $\% 20$ Thing.pdf $>$. Acesso em: dezembro 2019.

[21] SHASHANK, A. ; VIEIRA, D. “A survay on Internet of Things". Abakós, v. 01, n. 02. p. 78 a 95. ISSN: 23169451. Maio de 2013. Disponivel em: <file://C:/Users/ander/Downloads/5372-

Texto\%20do\%20artigo-21081-1-10-20130621.pdf>.

Acesso em: março 2020.

[22] SANTOS, B. P.; SILVA, L. A. M.; CELES, C. S. F. S.; BORGES NETO, J. B.; PERES, B. S.; VIEIRA, M. A. M.; VIEIRA, L. F. M.; GOUSSEVSKAIA, O. N.; LOUREIRO, A. A. F. "Internet das Coisas: da Teoria à Prática". Departamento de Ciência da Computação Universidade Federal de Minas Gerais -UFMG, Belo Horizonte - Minas Gerais.

[23] MATTERN, F; FLOERKEMEIER, C. "From the Internet of Computers to the Internet of Things". Distributed Systems Group - Institute for Pervasive Computing, ETH Zurich. DOI: 10.1007/978-3-642-172267_15

[24] ALMEIDA, P. S.; “Indústria 4.0: Princípios básicos, aplicabilidade e implantação na área industrial”, 2019. Editora Érica. ISBN 9788536530468

[25] ZANNI, A. "Sistemas cyber-físicos e cidades inteligentes". IBM- Developer Works, 2015. Disponível em: < https://www.ibm.com/developerworks/br/library/bacyber-physical-systems-and-smart-cities-iot/index.html>.

Acesso em: março 2020.

[26] LEE, J.; BAGHERI, B.; KAO, H. A. "Cyber Physical Systems architecture for Industry 4.0 - based manufacturing systems". ScienceDirect, 2014. DOI: 10.1016/j.mfglet.2014.12.001

[27] WINTER, J. "Sistemas Cyber Físicos: a nova revolução". Disponível em: $<$ https://www.eaware.com.br/sistemas-ciber-fisicos-a-novarevolucao/>. Setembro 2016. Acesso em: abril de 2020.

[28] BAGHERI, B.; YANG, S.; KAO, H.; LEE, J. “Cyber - physical Systems Architecture for Self - Aware Machines in Industry 4.0 Environment". ScienceDirect, 2015. DOI: 10.1016/j.ifacol.2015.06.318. 
[29] PISCHING, M. A.; TASCA, A. A.; PESSOA, M. A. $\mathrm{O}$; JUNQUEIRA, $\mathrm{F}$. et al. "Arquitetura para desenvolvimento de sistemas ciber-físicos aplicados na indústria 4.0". XIII Simpósio Brasileiro de Automação Inteligente, Porto Alegre, 2017. Disponível em: $<$ https://www.researchgate.net/publication/320394110_Arq uitetura_para_desenvolvimento_de_sistemas_ciber-

fisicos_aplicados_na_Industria $40>$. Acesso em: abril de 2020 .

[30] CROUÉ, N. "La fabrication additive : un nouvel paradigme dans la production". Essais \& simulation, MRJ Presse. n 124, p. 16-17. Março / abril de 2016.
[31] UZANU, J. "Essais et caractérisation de pièces en fabrication additive". Essais \& simulation, MRJ Presse. $n$ 124, p. 18-19. Março / abril de 2016.

[32] PISCOPO, M. R.; KNIESS, C. T.; TEIXEIRA, C. E.; BIANCOLINO, C. A. "O Setor Brasileiro de Nanotecnologia: Oportunidades e Desafios". XXXVII Encontro do ANPAD, 7 a 11 set. 2013. Rio de Janeiro. Anais..., 2013. 